\title{
Hierarchical Signal Processing for Tractable Power Flow Management in Electric Grid Networks
}

\author{
Pirathayini Srikantha, Member, IEEE, and Deepa Kundur, Fellow, IEEE
}

\begin{abstract}
Rapid advancements in smart grid technologies have brought about the proliferation of intelligent and actuating power system components such as distributed generation, storage and smart appliance units. Capitalizing fully on the potential benefits of these systems for sustainable and economical power generation, management and delivery is currently a significant challenge due to issues of scalability, intermittency and heterogeneity of the associated networks. In particular, vertically-integrated and centralized power system management is no longer tractable for optimally coordinating these diverse devices at large scale while also accounting for the underlying complex physical grid constraints. To address these challenges, we propose a hierarchical signal processing framework for optimal power flow management whereby the cyber-physical network relationships of the modern grid are leveraged to enable intelligent decision-making by individual devices based on local constraints and external information. Decentralized and distributed techniques based on convex optimization and game theoretic constructs are employed for information exchanges and decision-making at each tier of the proposed framework. It is shown via theoretical and simulation studies that our technique allows for the seamless integration of power components into the grid with low computational and communication overhead while maintaining optimal, sustainable and feasible grid operations.
\end{abstract}

\section{INTRODUCTION}

The rapidly evolving nature of the power grid today has transformed it into a highly diverse and dynamic network [1]. For example, the recent cyber-enablement of power system components, the rising penetration of sustainable Distributed Generation (DG) sources, the commercialization of smart appliances and the introduction of competitive energy markets promote a broad spectrum of extended grid capabilities such as advanced monitoring/control and sustainable operations [38], [39]. However, several open challenges deter full realization of such functionalities.

The main difficulties stem from the lack of compatibility of modern power devices with aging legacy infrastructure that still comprises a major part of the physical grid. For instance, the Distribution Network (DN), by design, transports power from the bulk grid to individual consumers. Now, with significant penetration of DGs (e.g. solar roof-top panels, etc.) at close proximity to consumers, power flow can conceivably occur in the reverse direction during excess generation periods. As distribution lines are associated with low reactance to

This work was supported in part by grants from the National Science Foundation and the Natural Sciences and Engineering Research Council of Canada. P. Srikantha is with the Department of Electrical and Computer Engineering at Western University and D. Kundur is with the Department of Electrical and Computer Engineering at University of Toronto, Email: psrikan@uwo.ca,dkundur@ece.utoronto.ca. resistance ratio, voltage rise is more substantial and excessive reverse power flow can then result in adverse equipment damages [2].

Another challenge is due to the vertically-integrated grid management paradigm (still utilized by many system operators). Competitive energy markets resulting from deregulation have spurred the deployment of private Independent Power Plants (IPPs). Directly controlling an immense number of these intermittent components in a centralized manner, as typical in vertically-integrated processes, is costly and unscalable. Without coordination in place, increasing congestion is evident in the Transmission Network (TN) which transports bulk power across the grid with lines operating close to maximum limits [3]. On the other hand, upgrading the infrastructure to accommodate these changes will result in exorbitant costs. Hence, it is necessary to employ alternative strategies to overcome these issues.

In this paper, we propose a hierarchical network-based signal processing framework that harnesses the intelligence and communication capabilities of actuating nodes to automate decision-making based on external information and local feasibility constraints. The structural characteristics of physical power networks residing at the transmission and distribution levels along with the highly granular monitoring information continuously generated in the grid are leveraged in the design of signals exchanged amongst these cyber-enabled nodes. These signals encapsulate general trends in the system which allow individual actuating nodes to adaptively and optimally respond to dynamically occurring changes while being attuned to local grid constraints and feasibility requirements. Abstraction and decoupling built into each tier of the proposed hierarchical system allows for the plug-and-play integration of a large number of diverse entities across TN and DN levels of the power grid in an optimal manner.

As such, our contributions are four-fold in this paper: 1) A novel hierarchical signal processing paradigm is presented along with a literature survey in this area; 2) A proof-ofconcept of how this framework can be effectively utilized for coordination over short horizons in the electric grid at the transmission and distribution network levels is presented using convex optimization [31] and game theoretic techniques [32]; 3) Design of signals, information exchange paradigm and actuation decisions are presented for both the TN and DN levels; and 4) Results from theoretical performance analysis and practical simulations are included along with comparison to existing state-of-the art. This paper is organized as follows. The Optimal Power Flow (OPF) formulation used 
by system operators for coordinating the grid is presented in Sec. [II along with a discussion of the associated challenges. This section is concluded with a literature survey. In Sec. III] the hierarchical model is presented. In Sec. IV and V decentralized and distributed coordination at the TN and DN levels are discussed. Mathematical tractability of the proposed framework is discussed in Sec. VI Simulation results are presented in Sec. VII Conclusions follow in Sec. VIII

\section{Optimal Power Flow and Challenges}

The Optimal Power Flow (OPF) formulation is typically constructed by the system operators to compute the minimal cost configuration of participating active nodes (e.g. power consumers, DGs, etc.) that are subject to power flow, bus voltage, power demand and generation constraints [4]. The most general form of the OPF problem is listed in $\mathcal{P}_{O P F}$ which is applicable to both TNs and DNs:

$$
\begin{aligned}
& \mathcal{P}_{O P F}: \min _{P, Q,|V|, \Theta} \sum_{i \in \mathcal{B}}\left(f_{i}\left(p_{i}^{g}\right)+f_{i}\left(q_{i}^{g}\right)+f_{i}\left(p_{i}^{r}\right)\right) \\
& \forall i \in \mathcal{B} \\
& p_{i}=p_{i}^{g}-p_{i}^{d}+p_{i}^{r}, q_{i}=q_{i}^{g}-q_{i}^{d}(\mathrm{C} 0) \\
& p_{i}=\sum_{j \in \mathcal{N}_{i}, i}\left|V_{i}\right|\left|V_{j}\right|\left(g_{i, j} \cos \left(\theta_{i}-\theta_{j}\right)+b_{i, j} \sin \left(\theta_{i}-\theta_{j}\right)\right)(\mathrm{C} 1) \\
& q_{i}=\sum_{j \in \mathcal{N}_{i}, i}\left|V_{i}\right|\left|V_{j}\right|\left(g_{i, j} \sin \left(\theta_{i}-\theta_{j}\right)-b_{i, j} \cos \left(\theta_{i}-\theta_{j}\right)\right)(\mathrm{C} 2) \\
& \underline{p}_{i}^{g} \leq p_{i}^{g} \leq \bar{p}_{i}^{g}, \underline{q}_{i}^{g} \leq q_{i}^{g} \leq \bar{q}_{i}^{g}, \underline{p}_{i}^{r} \leq p_{i}^{r} \leq \bar{p}_{i}^{r}, \underline{V}_{i} \leq\left|V_{i}\right| \leq \bar{V}_{i}(\mathrm{C} 3)
\end{aligned}
$$

The OPF problem is constructed on a physical network graph defined by buses $\mathcal{B}$, generators $\mathcal{G}$ and admittances (i.e. weight of each line). The optimization variables of $\mathcal{P}_{O P F}$ consist of the real and reactive power injections along with bus voltage magnitudes and angles which are represented by vectors $P \in \mathbb{R}^{|\mathcal{B}|}, Q \in \mathbb{R}^{|\mathcal{B}|},|V| \in \mathbb{R}^{|\mathcal{B}|}$ and $\Theta \in \mathbb{R}^{|\mathcal{B}|}$ respectively. Real power injection $p_{i} \in P$ at each Bus $i \in \mathcal{B}$ is further decomposed into three parts: power injected by local generators $p_{i}^{g}$, actual bus power demand $p_{i}^{d}$ and power demand reduction by flexible local consumers $p_{i}^{r}$. These result in $p_{i}=p_{i}^{g}-p_{i}^{d}+p_{i}^{r}$ where $p_{i} \in P$. Similarly, reactive power injection at Bus $i \in \mathcal{B}$ is defined as $q_{i}=q_{i}^{g}-q_{i}^{d}$ where $q_{i} \in Q$. The objective of $\mathcal{P}_{O P F}$ is a summation of functions operating on real and reactive power generation at each bus and demand reduction by flexible consumers.

Line $(i, j)$ connects Buses $i$ and $j$ and transports power between these. The associated bus admittance $y_{i, j}$ of $(i, j)$ is defined by conductance $g_{i, j}$ and susceptance $b_{i, j}$ parameters which capture power transfer properties across Bus $i$ and Bus $j$. The set $\mathcal{N}_{i}$ is composed of all buses that are directly connected to Bus $i$ via a single line. Constraints 1 and 2 enforce power balance at each bus for real and reactive power injections. Capacity constraints and voltage stability requirements impose upper and lower limits on variables $p_{i}^{g}, p_{i}^{d}, p_{i}^{r}$ and $V_{i}$. Upper limits are denoted by parameters $\bar{p}_{i}^{g}, \bar{p}_{i}^{d}, \bar{p}_{i}^{r}$ and $\bar{V}_{i}$ respectively. Similarly, lower limits are represented by the parameters $\underline{p}_{i}^{g}, \underline{p}_{i}^{d}, \underline{p}_{i}^{r}$ and $\underline{V}_{i}$ respectively. A realistic power system consists of a large number of buses that are associated with highly fluctuating parameters and variables. Efficient processing of information and tailored communications are necessary for optimal actuation which is the focus of this paper.

\section{A. Challenges}

The main difficulty in directly solving $\mathcal{P}_{O P F}$ stems from constraints (C1) and (C2) (derived from Kirchhoff's voltage and current laws) representing the net real and reactive power injections into Bus $i$. Quadratic and sinusoidal terms contribute to the non-convexity of these equality constraints. An optimization problem consisting of non-convex constraints is classified as NP-Hard. Problems falling in this class cannot be solved in a tractable manner especially for large systems [5]. Moreover, the underlying structure of the graph representing the physical system is a large and complex network consisting of mixed topologies (i.e. mesh and/or tree) and significant coupling for which analysis is not straightforward.

These difficulties are further exacerbated with the blurring of traditionally well-defined roles associated with power consumers and generators. Consumers are now actively generating sustainable power via roof-top solar panel and micro-wind turbine DGs. Power loads are no longer static as consumers have inherent demand flexibility that can be effectively leveraged using Energy Management Systems (EMSs) to promote sustainable energy consumption. Deregulation has enabled privately managed IPPs to competitively participate in active generation. This movement has introduced a large number of optimization variables and highly fluctuating parameters that have led to significant unpredictability in the grid and frequent system congestions.

\section{B. Literature Review}

Existing literature pertaining to optimal power flow management is extensive. Generally, these techniques apply some form of simplification to the original OPF listed in $\mathcal{P}_{O P F}$ to overcome the challenges outlined above. The simplified problem is then solved using one of centralized, decentralized and hierarchical grid network management techniques. This hierarchical signal processing technique essentially summarizes the pertinent information about lower tiers and abstract unnecessary details which will otherwise add significant complexity to the actuation process.

1) OPF Simplification Techniques: The simplest approach in the literature entails completely eliminating the problematic constraints from the optimization formulation [6]. As these constraints capture the underlying physical grid properties, ignoring these will not be appropriate for real-time power supply and demand matching in today's aggressively utilized power networks. Linearization methods are also investigated widely in the literature. A commonly used technique applies a series of simplifications to eliminate reactive power flows and bus voltage magnitudes from the OPF. This method also excludes important variables capturing the underlying physical system attributes. Other related work such as reference [7] applies double approximation where the cosine terms in the power balance equations are first replaced with quadratic terms. Then, 
another relaxation is applied to the resulting quadratic equality constraints. The resulting formulation consists of quadratic inequality constraints that are convex but computationally expensive than linear constraints. Reference [8] linearizes these quadratic terms via the loss factor-based linearization method based on sensitivity analysis. Other relaxation techniques such as cone and semi-definite relaxations are applied instead to obtain a tight convex representation of the original feasible set formed by $(\mathrm{C} 1)-(\mathrm{C} 3)$ [4]. These result in exact solutions for radial power networks (e.g. DNs) under some mild conditions [9]. These conditions, however, fail in mesh topologies which typically represent TNs [10]. To render convex relaxations exact in these systems, the authors of [11] propose the strategic addition of Flexible Alternating Current Transmission System (FACTS) devices. As these devices are costly, this is an expensive approach. Another option involves applying heuristics which are commonly used to solve non-convex optimization problems [12]. These provide no guarantee with respect to the convergence rate and optimality of the computed solution.

2) Centralized Strategies: Centralized grid management involves the computation of optimal operating setpoints of actuating grid entities by the system operator over long time horizons (e.g. once a day or every hour) using the original or simplified version of the OPF [4]. Forecast models are leveraged to compute system parameters (e.g. generation capacities and demands) over these extended periods. These models are however associated with significant error margins especially due to the presence of renewable resources and highly fluctuating power consumers in the system [13]. Smaller the time horizon considered, the lower is the associated error of the prediction algorithms. However, with smaller coordination horizons, the system operator is subject to significant computational and communication overheads entailed in frequently solving the selected OPF for a large number of optimization variables.

3) Decentralized Strategies: In decentralized strategies proposed in the literature, every actuating element typically participates in iteratively computing the optimal solution. Communication signals are typically exchanged between neighbouring nodes only. Thus, the central point of control is completely eliminated in this grid coordination paradigm. Methods that include dual decomposition, consensus and monotone operators are applied to a relaxed version of the OPF to compute the iterative decision-making by participating nodes [6], [14], [15], [39]. As the convergence rates of these strategies are proportional to the number of agents participating in the coordination process, greater the number of agents coordinated the more time is required for convergence. Other coordination mechanisms that are based purely on local measurements are myopic and examples include droop control employed by generation systems to adjust to instantaneous fluctuations in frequencies and power consumption [3]. Although control at this level addresses transient disturbances, these are not designed for optimality due to limitations in the information available.
4) Hierarchical Strategies: As managing power flow across the entire grid at high granularity is an intractable task, proposals such as reference [17] have divided the coordination process into general tiers such as tertiary, secondary and primary control layers. The computational methods evoked at each layer are based on either centralized or decentralized methods outlined in the above. For instance, in practical settings, coordination at the tertiary level entails planning and for this only real power balance is considered (i.e. reactive power and voltages are ignored). The control horizon in this tier can be as long as one day and central optimization is utilized here. In the secondary tier, central computations are conducted over hourly intervals using dynamic security assessment to compute the operating setpoints of generation systems [18]. In the primary control level, droop control mechanisms are utilized by large synchronous generation sources to automatically adapt to fluctuations. The afore-mentioned hierarchical coordination system is ideal for a grid consisting of predictable generation systems and power consumers. This is, however, no longer the case as today's grid consists of a large number of highly fluctuating actuating entities. Recent work on hierarchical approaches for power flow management utilize constructs such as clustering and partitioning to decompose the power grid into regions consisting of power elements that have significant coupling amongst one another [19], [20]. Local coordination is conducted within these partitioned regions and then combined in the subsequent tier. As the grid topology is no longer static due to the presence of renewable generation, electric vehicles and smart breakers, these static analyses may not account for the dynamic nature of today's grid. Other hierarchical methods proposed for managing small microgrid communities cannot be applied to manage an entire power grid [21], [22].

\section{HierarchicAl Model}

There are three main challenges associated with optimally coordinating the modern power grid:

1) Presence of a large number of optimization variables and non-convex constraints;

2) Unpredictability of fluctuating actuating elements; and

3) Congestions due to power infrastructure limitations.

Effectively overcoming these issues entails the incorporation of abstraction and independent decision-making. For this, we leverage a hierarchical steady-state power management approach illustrated in Fig. 1 composed of three tiers. These three tiers are inspired by the primary, secondary and tertiary grid management paradigms commonly adopted by grid operators [4]. Main differences between the proposed and existing frameworks stem from the granularity of coordination, signals exchanged between actuating entities and incorporation of physical power flow constraints. Decision-making at every tier by each node depends only on:

1) Local aggregate conditions of components managed in the subsequent tier; and

2) External information encapsulating the general state of the system with respect to optimality. 


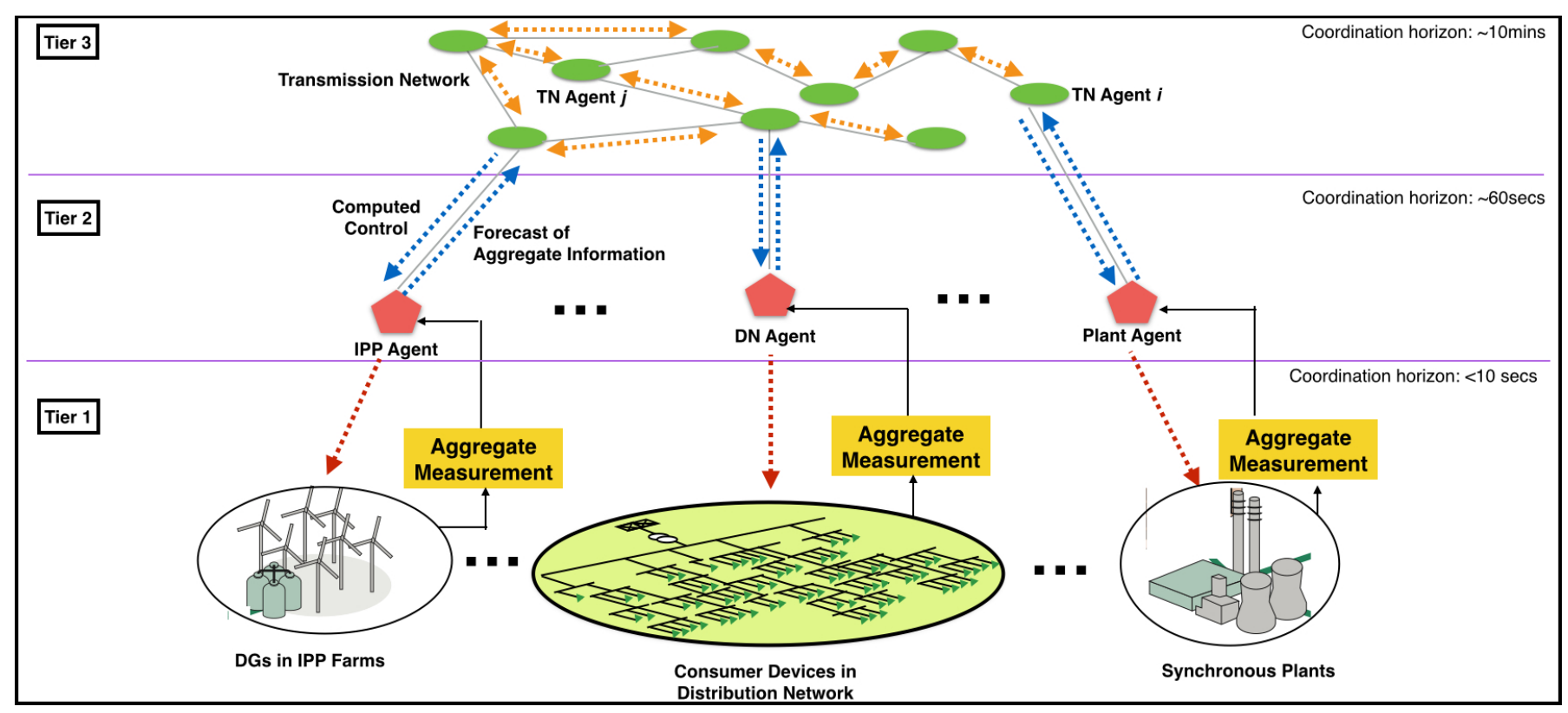

Fig. 1: Hierarchical Power Flow Management.

TABLE I: Hierarchical Coordination Summary.

\begin{tabular}{|l|l|l|l|}
\hline Tier & Nodes & Communication Signals Received & Communication Signals Sent \\
\hline Tier 1 & Consumer Loads & Receive general signals from DN agent & $\begin{array}{l}\text { Send local appliance control signals based on appliance } \\
\text { state and consumer comfort levels }\end{array}$ \\
\hline & DGs & $\begin{array}{l}\text { Receive general signals from IPP agent (for generation farms) } \\
\text { or DN agent (for micro-generation in consumer homes) }\end{array}$ & $\begin{array}{l}\text { Send local actuation signals depending on local } \\
\text { generation capacities }\end{array}$ \\
\hline & Synchronous Plants & Receive general signals from plant agent & $\begin{array}{l}\text { Send local actuation signals based on local } \\
\text { generation capacities }\end{array}$ \\
\hline Tier 2 & $\begin{array}{l}\text { IPP agent, DN agent, } \\
\text { or Plant agent }\end{array}$ & Receive general signal from managing entity in Tier 3 & $\begin{array}{l}\text { Aggregate local measurements and communicate } \\
\text { this to managing entity in Tier 2 }\end{array}$ \\
\hline Tier 3 & $\begin{array}{l}\text { TN agent } \\
\text { Receive general forecast signal from local agents in Tier 2 } \\
\text { and receive information from neighbouring TN agents }\end{array}$ & $\begin{array}{l}\text { Send general information to neighbouring TN agents } \\
\text { for iteratively computing optimal local power injection }\end{array}$ \\
\hline
\end{tabular}

The large grid coordination problem is now divided into manageable sub-problems and details of external entities are hidden. Hence, the number of variables handled by each node is a small subset of the original problem. The coordination horizon considered at each tier is divided into various scales ranging from a few minutes to seconds with granularity increasing from top to bottom of the hierarchy. This division of the coordination horizon reduces error margins associated with using prediction models over long forecast periods. Moreover, nodes at each tier utilize communication paradigms that are uniquely suited for the underlying physical requirements and associated resource constraints. Table II presents a summary coordination exacted across the three tiers with details listed in the following.

\section{A. Coordination at Tier 1}

The lowest tier (Tier 1) consists of individual power consumers, Independent Power Plants consisting of renewable generation and synchronous generation systems. It is important to note that coordination at this tier is local to individual consumer and generation entities. The coordinating agents consist of EMSs that reside within consumer premises and power controllers in generation systems. These agents residing in Tier 1 will optimally actuate based on local feasibility (e.g. consumer comfort, local appliance statuses, local feeder bus voltages, and generation capacities) and the general signal broadcast by the corresponding managing entity (e.g. IPP Agent, DN Agent, Plant Agent) in Tier 2. To determine local feasibility, if there is more than one node in the system (e.g. multiple wind turbines in wind farms, consumers in DNs), nodes will communicate with one another as necessary within their local system (e.g. local elements in the wind farm, local consumers in the DN). The time scale for coordination is in the range of seconds to accommodate for the constant flux in operating conditions of local loads and generation entities. The managing entity in Tier 2 will broadcast a general signal every one second and local aggregate measurements are reported to the managing entity every one second. As point-to-point communication with individualized signals is not established by the managing entity to every consumer and generation system, computational and communication overheads are significantly reduced. Aggregate measurements can be readily reported by local data concentrators commonly present in the grid as part of the Advanced Metering Infrastructure (AMI) by aggregating sensor measurements generated by abundantly 
deployed Phasor Measurement Units (PMUs) [4].

\section{B. Coordination at Tier 2}

Tier 2 consists of agents (e.g. IPP, DN or Plant agents) representing individual systems such as synchronous generation plants, IPPs and DNs that directly connect to buses interfacing with the TN. Hence, there can be multiple IPP, DN and Plant agents in Tier 2 (depending on the number of these systems present in the grid). These agents manage the overall power injection of the individual systems that are directly coordinated by these. For example, power injections by synchronous generation plants and IPPs are managed by the associated individual plant authorities whose control entities serve as the Plant or IPP agents. Real power injection by a DN is indirectly managed by the associated Electric Power Utility (EPU) which serves as a DN agent. These agents will iteratively compute general signals every second and broadcast these to their associated control elements using general signals received from Tier 3 and aggregate information conveyed from Tier 1. The main objective of these agents is to coordinate the aggregate behaviour of their local systems within a control horizon of 60 seconds based on the requirements conveyed by the general signals relayed from Tier 3 . The general signals relayed from Tier 3 are transmitted every ten minutes and the Tier 2 agents will reformulate their optimization problem every ten minutes based on these general signals. Information is not exchanged between agents residing in Tier 2 . Tier 2 agents will also forecast aggregate parameters of the Tier 1 system (e.g. wind farm, DN) at every ten minute interval based on information relayed by the data concentrators in Tier 1 and send this to the managing entity in Tier 3. Since the forecast interval is very short (i.e. ten minutes) and is for individual systems composed of small number of entities, error margins will be very low.

\section{Coordination at Tier 3}

Tier 3 consists of agents representing each bus in the TN. TN agent $i$ representing Bus $i$, communicates with TN agents representing neighbouring buses to iteratively compute net real power injection $p_{i}$ in an iterative manner based on ten minute forecasts of demand/supply sent by local agents in Tier 2 and neighbouring $\mathrm{TN}$ constraints in Tier 3. Information exchange between neighbouring buses takes place every 10 milliseconds (to account for latencies in communication) [1] so that all the TN agents will converge to an optimal equilibrium within ten minutes. This optimal power injection computed by every TN agent is then relayed to local Tier 2 agents (i.e. DN, IPP and/or Plant agents). The coordination horizon of ten minutes in Tier 3 is significantly more granular than day-ahead unit commitment which is typically engaged for coordination at the TN level [4]. This granularity enables active and adaptive response by active nodes to short-term volatilities in the TN. Moreover, new TN nodes can be added to the system in a plug-and-play manner as there is no overhead associated with centralized administration. This model is well-suited for today's evolving landscape of competitive markets and deregulations.

\section{Summary}

The hierarchical signal processing and actuation setup summarized in the above allows for the complete abstraction of individual power component operations in a complex power network from a system-wide controller. At each tier, the agents utilize aggregate information and signals exchanged with neighbouring or managing entities to make local decisions. This markedly reduces communication overhead otherwise needed to forge individual communication links with all actuating nodes. Moreover, as computations are divided and parallelized amongst tiers and nodes, computational overhead is also greatly diminished. Although hierarchical management is not a unique concept (e.g. reference [6]), our work is a novel departure due to the granularity of optimization, scalability and the integration of various communication topologies based on the underlying physical constraints of the system. The primary, secondary and tertiary (PST) grid management paradigm utilized widely today to coordinate grid operations has some parallels to Tiers 1,2 and 3 respectively in the proposed hierarchical framework [4]. One common feature of these frameworks is the manner in which entities are organized within each levels. This is essentially based on the ownership and management structure of power system. For instance, in the PST framework, EPUs are organized based on geographical contexts and deliver power to directly to consumers. System operators deal with balancing power demand with supply at the TN level.

However, there are some marked differences. At the tertiary control level, EPUs purchase power from system operators based on day-ahead markets. Thus, the system operators utilize forecasts of demand and supply over very large time horizons (i.e. 24 hour period). In the secondary layer, spot-markets deal with hourly changes and the associated problem formulations incorporate only real power balance and bus angles. Reactive power and bus voltage magnitudes are ignored. Generation setpoints are computed and communicated to generators at hourly intervals which are maintained at the primary level. Controllers in individual generators adapt to instantaneous fluctuations in power demands by utilizing droop control techniques to maintain the system frequency at nominal levels and this is entirely based on local information. The tertiary layer results in significant error margins due to forecast errors, the secondary layer neglects important grid variables such as reactive power and bus voltage magnitudes that will lead to inefficiencies and finally the primary level leads to completely myopic decisions that do not account for the conditions of other entities in the system. In order to ensure that the system functions within acceptable stability limits in the event of unexpected events, contingency analyses are conducted by system operators and these cannot effectively account for fluctuations inherent in DGs. Moreover, actuation of flexible consumers is not included in this model.

In our framework, we incorporate power flow constraints 
in all tiers. In Tier 3, TN nodes exchange information with neighbours to compute physically viable setpoints. In Tier 1 , DN nodes exchange information with neighbouring nodes to ensure that the impending switch satisfies physical constraints. Tier 2 bridges Tier 3 and Tier 1 via generalized broadcast signals. The next differentiating attribute is that the largest coordination horizon is associated with Tier 3 which is ten minutes in length. This results in a significant reduction of error/inefficiencies due to forecast uncertainties.

\section{E. Proof-of-Concept}

Next, as a proof-of-concept, we will demonstrate in the remainder of this paper how the hierarchical signal processing model outlined in the above can be applied for practical coordination in the power grid. For Tier 3 coordination, we adopt the OPF formulation introduced in reference [23] which applies a series of transformations to obtain a close approximation of the original non-convex power flow constraints via a set of linear equality and inequality constraints without eliminating important features of the problem such as reactive power flow and bus voltages in the TN. Although the number of constraints will increase with this technique, these linear constraints capture the basic nuances of non-convex terms up to a fairly high level of accuracy and allow for greater computational efficiency [23]. Moreover, this technique has been recommended in references such as [10] as a viable alternative for modelling the TN. The main difference between our representation of the approximated power flow constraints and that proposed in [23] is our use of bus admittances instead of line admittances. We assert that bus admittances allow for the incorporation of realistic effects on power flow due to transformers, shunt admittances and line charging susceptances. We then design decentralized coordination for Tier 3 by applying ADMM to the afore-mentioned OPF model. ADMM is typically applied to relaxed OPF formulations associated with computational overheads stemming from the presence of quadratic inequality constraints constructed for the DN with radial connection topology (e.g. [15]) or non-convex OPF (e.g. [24]) which may not converge. Our novel contribution in the design of decentralized Tier 3 coordination is that we construct the optimal coordination problem at the transmission network level so that it is decomposable and separable at each actuating node even in the presence of cycles/loops which are typical in mesh networks while accounting for reactive power and bus voltage magnitude constraints. Signals designed using ADMM now encapsulate trends from the perspective of every cyber-enabled node arranged in a mesh configuration regarding optimality and feasibility in the transmission network. These signals that are generated and exchanged amongst actuating nodes are used to iteratively improve local actuation. These iterative revisions converge to the optimal solution even in the presence of loops due to the convexity of the decomposed problem. Moreover, important grid characteristics are retained in the local decision-making process. Accuracy of solutions in practical Tier 3 systems obtained via our method are presented in Sec. VII
For Tier 2 coordination, we utilize decomposition and population game theory to design general signals that will be utilized by nodes located in the DN, IPPs and/or large scale plants to make local actuation decisions. In the literature, distributed methods based on sub-gradient, consensus and game theoretic approaches have been proposed (e.g. [14], [25], [26]). Main challenges associated with these are the speed of convergence and scalability. As per our discussion on Tier 2 coordination, a completely decentralized solution is not necessary as aggregate measurements can be utilized to construct general signals that guide individual entities in Tier 1. We utilize population game theory for designing general signals. The incremental impact of actuation by individual active nodes enables the application of population game theory in designing generalized broadcast signals that iteratively guide these nodes with minimizing the system cost in a distributed manner. The dynamics resulting from these strategy revisions are guaranteed to converge to the optimal configuration. Local feasibility checks by the revising node ensure that DN bus voltage and apparent power flow constraints are met by impending changes in actuation. This differs from our earlier work in [27], [28] as we design signals based on Tier 3 and aggregate conditions in Tier 1. Response by individual elements in Tier 1 is based on signals transmitted from Tier 2 and local feasibility conditions.

In summary, the proposed signal processing framework allows for the coordination of diverse actuating elements residing in the complex and highly coupled electrical network. Structural features of the underlying physical networks along with highly granular monitoring information generated in the grid are leveraged to design and refine signals constructed and exchanged in the power system across the transmission, distribution and local actuation tiers.

\section{COORDINATION IN TIER 3}

The design of decentralized information exchange between TN agents for iteratively determining optimal power injection in Tier 3 where agents are arranged in a mesh topology as typical in the $\mathrm{TN}$ is detailed in the following.

\section{A. TN Line Characteristics}

Main attributes of the TN that affect power flow are line admittances $y_{i, j}^{l}=g_{i, j}^{l}+\mathbf{i} b_{i, j}^{l}$, line charging susceptances $y_{i, j}^{c}$, shunt admittances $y_{i, i}^{s}$ and transformer tap ratios $\left|T_{i, j}\right|$ and phase shifts $s_{i, j}$ (i.e. $T_{i, j}=\left|T_{i, j}\right| \angle s_{i, j}^{\circ}$ ) which are combined to form the Y-bus matrix consisting of the following elements:

$$
y_{i, i}=\sum_{j \in \mathcal{N}_{i}} \frac{2 y_{i, j}^{l}+y_{i, j}^{c}}{2\left|T_{i, j}\right|^{2}}+y_{i, i}^{s}, \quad y_{i, j}=\frac{-y_{i, j}^{l}}{\mathbb{I}_{i, j} T_{i, j}^{*}+\mathbb{I}_{j, i} T_{i, j}}
$$

Set $\mathcal{N}_{i}$ consists of all buses that are adjacent to Bus $i$ (i.e. directly connected to Bus $i$ via a line) and $\mathbb{I}_{i, j}$ is an indicator function that returns 1 if line $i \leftrightarrow j$ is in the direction of stepped-down voltage and 0 otherwise. $\left|T_{i, j}\right|$ and $s_{i, j}$ for lines without transformers are set to 1 and 0 respectively. Each element of the $Y$-bus matrix $y_{i, j}$ can be separated into real and reactive components which are referred to as bus conductance $g_{i, j}$ and bus susceptance $b_{i, j}$ respectively. These line properties, connecting bus angles and voltages dictate the 
net real $\left(p_{i}\right)$ and reactive $\left(q_{i}\right)$ power injections into each Bus $i$ as listed in (C1) and (C2) of $\mathcal{P}_{O P F}$ and these are nonconvex constraints. The following approximations are applied to transform these into a set of convex constraints.

\section{B. Approximation of Power Flow Constraints}

First, consider the real power flow constraint (C1). Real power flow is influenced significantly by differences in bus angles rather than bus voltage magnitudes. For this reason, the voltage magnitude variables $\left|V_{i}\right|$ and $\left|V_{j}\right|$ are replaced by constants $\left|V_{i}^{t}\right|$ and $\left|V_{j}^{t}\right|$ that can either be bus voltage magnitude setpoints or the current voltage magnitude measured prior to a change in the system. This eliminates the voltage magnitude variables in (C1). Next, the $\sin \left(\theta_{i}-\theta_{j}\right)$ term is replaced by $\theta_{i}-\theta_{j}$ as the phase angle differences are very small (close to zero) and the sine function is approximately linear close to the origin. Finally, the cosine term is approximated by a set of linear inequality constraints as outlined in reference [23]. We do not apply first-order Taylor series expansion to approximate this function as the cosine term is not linear around the origin. A new variable $c \hat{o} s_{i, j}$ subject to the following $n+2$ constraints is introduced to replace the $\cos \left(\theta_{i}-\theta_{j}\right)$ term:

$$
\begin{aligned}
& \cos _{i, j} \geq 0 \\
& \cos _{i, j} \leq-\sin \left(a_{k}\right)\left(\theta_{i}-\theta_{j}-a_{k}\right)+\cos \left(a_{k}\right) \forall k \in n \\
& \cos _{i, j}=\cos s_{j, i}
\end{aligned}
$$

where $a_{k}=-\frac{\pi}{2}+\frac{\pi}{n+1} k$. The inequalities are linearly dependent on the bus angles $\theta_{i}$ and $\theta_{j}$. The third constraint ensures that the côs terms are the same for both directions of the line.

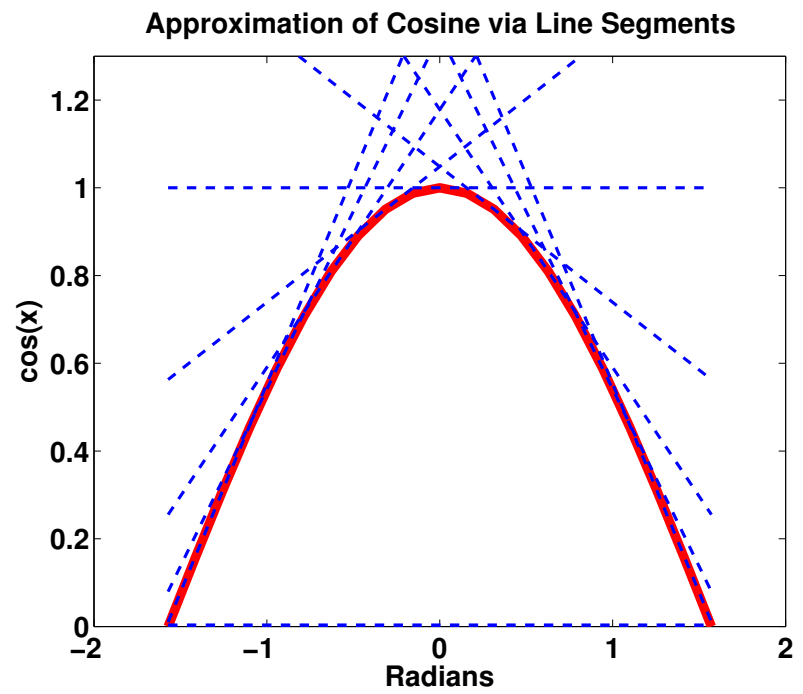

Fig. 2: Approximation of $\cos (\theta)$ via Linear Inequalities.

Fig. 2 illustrates how the positive region enclosed by the $\cos (\theta)$ function is approximated by (C1'-C3') when $n=9$. The maximum value $c \hat{o} s_{i, j}$ can take after being subjected to these inequalities results in a close approximation of $\cos \left(\theta_{i}-\theta_{j}\right)$. Hence, in the minimizing objective, an additional term $-c \hat{o} s_{i, j}$ is added to obtain this close approximation. Combining all of these steps results in:

$$
p_{i}^{g}+p_{i}^{r}-p_{i}^{d}=\sum_{j \in \mathcal{N}_{i}, i}\left|V_{i}^{t}\right|\left|V_{j}^{t}\right|\left(g_{i, j} c \hat{o} s_{i, j}+b_{i, j}\left(\theta_{i}-\theta_{j}\right)\right)\left(\mathrm{C}^{\prime}{ }^{\prime}\right)
$$

(C1')-(C4') along with the additional $-c \hat{o} s_{i, j}$ term in the objective form the linear approximation of $(\mathrm{C} 1)$.

Next, the reactive power flow constraint (C2) is linearized by applying another set of approximations. As reactive power flow significantly depends on changes in voltage magnitudes, it is necessary to take these into account in the approximation. To enable this, the bus voltage $\left|V_{i}\right|$ is separated into two components $\left|V_{i}\right|=\left|V_{i}^{t}\right|+\phi_{i} .\left|V_{i}^{t}\right|$ is the target voltage magnitude and $\phi_{i}$ represents the deviation of the actual voltage magnitude from the target. $\left|V_{i}^{t}\right|$ is known in advance and is therefore a constant. $\phi_{i}$ is a new variable. Substituting these into the quadratic voltage magnitude terms in $(\mathrm{C} 2)$ results in:

$$
\left|V_{i}\right|\left|V_{j}\right|=\left|V_{i}^{t}\right|\left|V_{j}^{t}\right|+\phi_{i}\left|V_{j}^{t}\right|+\phi_{j}\left|V_{i}^{t}\right|+\phi_{i} \phi_{j}
$$

The first term in the above relation is constant. This term multiplies into $g_{i, j} \sin \left(\theta_{i}-\theta_{j}\right)+b_{i, j} \cos \left(\theta_{i}-\theta_{j}\right)$. Applying the linear approximation of the sine and cosine terms results in $\left|V_{i}^{t}\right|\left|V_{j}^{t}\right|\left(g_{i, j}\left(\theta_{i}-\theta_{j}\right)+b_{i, j} c \hat{o} s_{i, j}\right)$. However, the remaining three terms are still problematic as these consist of non-linear components such as $\phi_{i}\left(\sin \left(\theta_{i}-\theta_{j}\right)\right)$, etc. First-order linear terms in the Taylor series expansion of these nonlinear terms replace these where $\phi_{i}, \phi_{j}$ and $\theta_{i}-\theta_{j}$ are evaluated around 0 as these are expected to be close to 0 . These changes result in the following linearized approximation of $(\mathrm{C} 2)$ :

$$
\begin{gathered}
q_{i}^{g}-q_{i}^{d}=\sum_{j \in \mathcal{N}_{i}, i}\left[\left|V_{i}^{t}\right|\left|V_{j}^{t}\right|\left(g_{i, j}\left(\theta_{i}-\theta_{j}\right)-b_{i, j} c \hat{o} s_{i, j}\right)\right. \\
\left.-\left(\phi_{i}\left|V_{j}^{t}\right|+\phi_{j}\left|V_{i}^{t}\right|\right) b_{i, j}\right]
\end{gathered}
$$

\section{Approximation Errors}

Three main approximations are made in the process outlined above. First, $\sin \left(\theta_{i}-\theta_{j}\right)$ is replaced with $\theta_{i}-\theta_{j}$. As the phase angle differences between TN buses are typically close to 0 , the linear term adequately approximates the sine term around the origin. Next, the cosine term is replaced with a new optimization variable $c \hat{o} s_{i, j}$ which is subject to linear inequality constraints. As evident in Fig. 2, when the number of line segments increases, the greater is the accuracy of representation of the cosine term. We utilize 21 line segments in our practical studies presented in Sec. VII to approximate the cosine term. The $-c \hat{o} s_{i, j}$ term is added to the objective function of the linearized OPF to obtain a close approximation of the original cosine function. Although this term can introduce an offset to the optimal value of the objective function, values taken by $c \hat{o} s_{i, j} \in[0,1]$ is negligible in comparison to the values taken by the original objective function $f$ of the OPF. Finally, the remaining non-linear terms in the power balance equations are approximated using the first-order Taylor series expansion around the origin with error that is quadratic (in the worst case) with respect to the deviation of independent variables from the origin. As this deviation is expected to be minimal, this error is inconsequential. 


\section{Separable Formulation}

After applying the aforementioned transformations, the original power flow equations can now be approximated by the linear constraints $\left(\mathrm{Cl}^{\prime}\right)-\left(\mathrm{C}^{\prime}\right) . \mathcal{P}_{O P F}^{c}$ is convex as all constraints are now linear and the objective is convex [4]:

$$
\begin{aligned}
& \mathcal{P}_{O P F}^{c}: \min _{P, Q, \Phi, \Theta, c o s s} \sum_{i \in \mathcal{B}}\left(f_{i}\left(p_{i}^{g}\right)+f_{i}\left(q_{i}^{g}\right)+f_{i}\left(p_{i}^{r}\right)\right)-\sum_{i \leftrightarrow j} c \hat{o} s_{i, j} \\
& \text { s.t. (C1') }-\left(\mathrm{C}^{\prime}\right),(\mathrm{C} 3), \forall i, j \in \mathcal{B}
\end{aligned}
$$

Although this problem can be solved exactly by commercially available solvers, it is difficult to centrally coordinate fluctuating renewable generation entities and consumer demands at a highly granular level while ensuring that the system remains within secure operational limits [29].

A decentralized approach, whereby all the active power injecting buses in the system iteratively contribute to the optimal solution by performing individual computations based on locally changing demand and generation requirements, will alleviate significant overhead otherwise incurred through central coordination. Moreover, this also allows every active participant to adapt to changes in the system in an automated and cost effective manner. The decentralized algorithm proposed in this paper depends on information exchanges between neighbouring buses. Hence, the OPF must be formulated so that it is separable amongst all the buses in the system and each TN agent representing a bus depends only on information updates sent by neighbouring buses to solve its local problem. Each TN agent performs iterative updates of its local solution by leveraging on ADMM. Convergence to optimality is guaranteed for this method as the problem is convex [30].

1) Background on ADMM: Next, a general overview of ADMM is presented. The method by which $\mathcal{P}_{O P F}^{c}$ is reformulated so that ADMM can be applied is discussed thereafter.

ADMM is an operator splitting method that can be applied to problems with the following general form [31]:

$$
\begin{gathered}
\mathcal{P}_{A D M M}: \min _{\substack{x \in \mathcal{X}, y \in \mathcal{Y} \\
A x=B y .}} f(x)+g(y) \\
\text { Ax }
\end{gathered}
$$

The optimization variables $x \in \mathbb{R}^{b}$ and $y \in \mathbb{R}^{c}$ are each subject to individual constraints represented by convex sets $\mathcal{X}$ and $\mathcal{Y}$. The objective is a summation of two terms that are convex functions of $x$ and $y$ respectively. These variables are coupled to each other by $p$ equality constraints that are succinctly represented by constant matrices $A \in \mathbb{R}^{p \times b}$ and $B \in \mathbb{R}^{p \times c}$. Without the equality constraints, $\mathcal{P}_{A D M M}$ would be completely separable and the optimal solutions $x^{*}, y^{*}$ can then be computed individually. In order to enable the decomposition of $\mathcal{P}_{A D M M}$, the augmented Lagrangian $L_{\rho}(x, y, \nu)$ is constructed as follows:

$$
\mathcal{L}_{\rho}(x, y, \nu)=f(x)+g(y)+\nu^{T}(A x-B y)+\frac{\rho}{2}\|A x-B y\|_{2}^{2}
$$

where the first two functions are from the objective in $\mathcal{P}_{A D M M}, \nu \in \mathbb{R}^{p}$ is the vector of Lagrangian multipliers associated with each equality constraint in $\mathcal{P}_{A D M M}$, the last term is the augmentation term that enforces strict convexity when $\rho>0 . \mathcal{L}_{\rho}(x, y, \nu)$ is used to update $x, y$, and $\nu$ individually in an iterative manner. At the $k^{t h}$ iteration, the following updates are applied in series:

$$
\begin{aligned}
x^{k+1} & =\underset{x}{\operatorname{argmin}} \mathcal{L}_{\rho}\left(x, y^{k}, \nu^{k}\right) \\
y^{k+1} & =\underset{y}{\operatorname{argmin}} \mathcal{L}_{\rho}\left(x^{k+1}, y, \nu^{k}\right) \\
\nu^{k+1} & =\nu^{k}+\rho\left(A x^{k+1}-B y^{k+1}\right)
\end{aligned}
$$

where the first update is applied to $x$ when $y$ and $\nu$ are fixed to values computed from the previous iteration. The next update is applied to $y$ where $x^{k+1}$ is the value computed immediately before and $\nu^{k}$ has been computed in the previous iteration. $\nu$ is a dual variable and is updated using a subgradient like method where $x$ and $y$ are set to $x^{k+1}$ and $y^{k+1}$ computed in the current iteration. It is evident that at each iteration, three sets of information exchanges are necessary to update the primal and dual variables in a decentralized manner.

2) Converting Convex OPF into the ADMM Form: $\mathcal{P}_{O P F}^{c}$ can also be converted into the form listed in $\mathcal{P}_{A D M M}$ to which decentralized updates outlined above can be applied by individual buses aided by local information exchanges. For this, new variables are first introduced that serve as perspectives of variables belonging to neighbouring buses and this notion of perspective variables was first introduced in [15]. Consensus is then established between these and the original variables. Our work differs vastly from reference [15] as we are applying ADMM at the TN level which is of a mesh topology that can consist of cycles whereas reference [15] applies ADMM to the DN which is a radial/tree structure with no loops. We consider the bus injection model to represent the network topology of the TN whereas reference [15] utilizes the branch-flow model to represent the DN. The constraints considered in this paper are based on linear approximations of the AC power balance constraints which is well-suited at the TN level [10]. On the other hand, reference [15] utilizes second-order cone relaxations which is well suited in the context of DN not TN. Thus, the problem formulation (e.g. variables and constraints), signals exchanged and the topology under consideration differs significantly from prior art.

Upon examining $\mathcal{P}_{O P F}^{c}$, it is clear that constraints $\left(\mathrm{Cl}^{\prime}\right)-$ (C5') associated with each bus depend on variables such as $\theta$ and $\phi$ of neighbouring buses. (C3) represents constraints that are completely local to Bus $i$ and therefore are separable. To render constraints $\left(\mathrm{C1}^{\prime}\right)-\left(\mathrm{C}^{\prime}\right)$ separable as well, new variables are introduced which will be referred to as perspective variables. TN agent $i$ will maintain a set $y_{i}$ consisting of variables which are local perspectives of certain optimization variables of local and neighbouring buses as follows:

$$
y_{i}=\left\{p_{i, i}^{g}{ }^{(y)}, p_{i, i}^{r}{ }^{(y)}, q_{i, i}^{g}{ }^{(y)}, \theta_{i, i}^{(y)}, \phi_{i, i}^{(y)}, \phi_{j, i}^{(y)}, \theta_{j, i}^{(y)}, c \hat{o} s_{i, j}^{(y)} \forall j \in \mathcal{N}_{i}\right\} .
$$

The first subscript index in each variable denotes the perspective of and the second index indicates the perspective from. For example, $\theta_{j, i}$ is the perspective of the voltage angle of Bus $j$ from Bus $i$. Perspectives of local variables are maintained in order to allow for complete separability of the $y$ variables. As TN agent $i$ will not have direct access to optimization variables of neighbouring nodes, perspective variables serve 
as local substitutes of the actual variables used in constraints $\left(\mathrm{C}^{\prime}\right)-\left(\mathrm{C}^{\prime}\right)$. Now, TN agent $i$ can evaluate these constraints locally. $\left(\mathrm{Cl}^{\prime}\right)-\left(\mathrm{C}^{\prime}\right)$ are reformulated with the perspective variables as follows which form the constraint set $\mathcal{Y}_{i}$ :

$-c \hat{o} s_{i, j}^{(y)} \leq 0$

$\cos _{i, j}^{(y)} \leq-\sin \left(a_{k}\right)\left(\theta_{i, i}^{(y)}-\theta_{j, i}^{(y)}-a_{k}\right)+\cos \left(a_{k}\right) \forall k \in n$

$p_{i, i}^{g(y)}+p_{i, i}^{g(y)}-p_{i, i}^{d}{ }^{(y)}=\sum_{j}\left|V_{i}^{t}\right|\left|V_{j}^{t}\right|\left(g_{i, j} c \hat{o} s_{i, j}+b_{i, j}\left(\theta_{i, i}^{(y)}-\theta_{j, i}^{(y)}\right)\right)$

$q_{i, i}^{g(y)}-q_{i, i}^{d(y)}=\sum_{j}\left[\left|V_{i}^{t}\right|\left|V_{j}^{t}\right|\left(g_{i, j}\left(\theta_{i, i}^{(y)}-\theta_{j, i}^{(y)}\right)-b_{i, j} c \hat{o} s_{i, j}\right)\right.$

$\left.-\left(\phi_{i, i}^{(y)}\left|V_{j}^{t}\right|+\phi_{j, i}^{(y)}\left|V_{i}^{t}\right|\right) b_{i, j}\right]$.

Next, the original optimization variables are maintained in set $x_{i}$ for each TN agent $i$ as follows:

$$
x_{i}=\left\{p_{i}^{g(x)}, p_{i}^{r(x)}, q_{i}^{g(x)}, \theta_{i}^{(x)}, \phi_{i}^{(x)}, c \hat{o} s_{i, j}^{(x)} \forall j \in \mathcal{N}_{i}\right\} .
$$

These are subject to the local separable constraints from (C3) which form the feasible set $\mathcal{X}_{i}$ :

$$
\begin{aligned}
& \underline{p}_{g}^{i} \leq p_{i}^{g(x)} \leq \bar{p}_{g}^{i}, \underline{p}_{r}^{i} \leq p_{i}^{r(x)} \leq \bar{p}_{r}^{i}, \underline{q}_{g}^{i} \leq q_{i}^{g(x)} \leq \bar{q}_{g}^{i}, \\
& \underline{V}_{i} \leq \phi_{i}^{(x)}+\left|V_{i}^{t}\right| \leq \bar{V}_{i}
\end{aligned}
$$

As a final step, it is necessary to establish a consensus between the perspective and original variables. For instance, the perspective variable $\theta_{j, i}^{(y)}$ must be equal to the original variable $\theta_{j}^{(x)}$. All the consensus constraints associated with TN agent $i$ are listed in the following:

$$
\begin{array}{lr}
p_{i}^{g(x)}=p_{i, i}^{g}{ }^{(y)}, p_{i}^{r(x)}=p_{i, i}^{r}{ }^{(y)}, q_{i}^{g(x)}=q_{i, i}^{g(y)} & \\
\theta_{i}^{(x)}=\theta_{i, j}^{(y)}, \phi_{i}^{(x)}=\phi_{i, j}^{(y)} & \forall j \in\left(\mathcal{N}_{i}, i\right) \\
\cos _{i, j}^{(x)}=c \hat{o} s_{j, i}^{(y)}, \cos _{j, i}^{(x)}=c \hat{o} s_{i, j}^{(y)} & \forall j \in \mathcal{N}_{i}
\end{array}
$$

which can be generally expressed as $A_{i} x_{i}=B_{i} y_{i}$. Combining all of the afore-mentioned transformations results in:

$$
\begin{gathered}
\mathcal{P}_{O P F}^{d}: \min _{x_{i} \in \mathcal{X}_{i}, y_{i} \in \mathcal{Y}_{i} \forall i \in \mathcal{B}} \sum_{i \in \mathcal{B}}\left(f_{i}\left(p_{i}^{g(x)}\right)+f_{i}\left(q_{i}^{g(x)}\right)+f_{i}\left(p_{i}^{r(x)}\right)\right) \\
A_{i} x_{i}=B_{i} y_{i} \forall i \in \mathcal{B}
\end{gathered}
$$

which is equivalent to the original formulation $\mathcal{P}_{O P F}^{c}$. It is also clear that this problem has the same structure as $\mathcal{P}_{A D M M}$. Hence, ADMM can now be applied to $\mathcal{P}_{O P F}^{d}$ for decentralized computation of local power injection by every active bus in the TN. First, the augmented Lagrangian is constructed:

$$
\begin{aligned}
& L_{\rho}(x, y, \nu)=\sum_{i \in \mathcal{B}}\left(f_{i}\left(p_{i}^{g(x)}\right)+f_{i}\left(q_{i}^{g(x)}\right)+\nu_{i, i}^{p}\left(p_{i}^{g(x)}-p_{i, i}^{g(y)}\right)+\right. \\
& \nu_{i, i}^{r}\left(p_{i}^{r(x)}-p_{i, i}^{r}{ }^{(y)}\right)+\nu_{i, i}^{q}\left(q_{i}^{g(x)}-q_{i, i}^{g(y)}\right)+\sum_{j \in \mathcal{N}_{i}}\left[\nu _ { i , j } ^ { c o s } \left(c \hat{o} s_{i, j}^{(x)}\right.\right. \\
& \left.\left.-c \hat{o} s_{i, j}^{(y)}\right)+\nu_{j, i}^{c o s}\left(c \hat{o} s_{i, j}^{(x)}-c \hat{o} s_{j, i}^{(y)}\right)\right]+\sum_{j \in \mathcal{N}_{i}, i}\left[\nu_{i, j}^{\theta}\left(\theta_{i}^{(x)}-\theta_{i, j}^{(y)}\right)+\right. \\
& \left.\nu_{i, j}^{\phi}\left(\phi_{i}^{(x)}-\phi_{i, j}^{(y)}\right)\right]+\frac{\rho}{2}\left(p_{i}^{g(x)}-p_{i, i}^{g}{ }^{(y)}\right)^{2}+\frac{\rho}{2}\left(q_{i}^{g(x)}-q_{i, i}^{g(y)}\right)^{2}+ \\
& \frac{\rho}{2}\left(p_{i}^{r(x)}-p_{i, i}^{r}{ }^{(y)}\right)^{2}+\frac{\rho}{2} \sum_{j \in \mathcal{N}_{i}, i}\left[\left(\theta_{i}^{(x)}-\theta_{i, j}^{(y)}\right)^{2}+\left(\phi_{i}^{(x)}-\phi_{i, j}^{(y)}\right)^{2}\right]+ \\
& \left.\frac{\rho}{2} \sum_{j \in \mathcal{N}_{i,}}\left[\left(c \hat{o s} s_{i, j}^{(x)}-\operatorname{coss}_{i, j}^{(y)}\right)^{2}+\left(c o s_{i, j}^{(x)}-c \hat{o} s_{j, i}^{(y)}\right)^{2}-2 c \hat{o s}{ }_{i, j}^{(x)} / \rho\right]\right) .
\end{aligned}
$$

It is evident from this construction that each TN agent $i$ is associated with a set of Lagrangian multipliers $\nu_{i}$ :

$$
\nu_{i}=\left\{\nu_{i, i}^{p}, \nu_{i, i}^{r}, \nu_{i, i}^{q}, \nu_{i, j}^{\theta}, \nu_{i, j}^{\phi}, \nu_{i, j}^{\text {cos }}, \nu_{j, i}^{\cos } \forall j \in \mathcal{N}_{i}\right\} .
$$

$\mathcal{L}_{\rho}(x, y, \nu)$ can now be decomposed into individual subproblems that can be solved by each bus. Iterative updates taking place every $0.1 \mathrm{~s}$ (communication delay is typically in the range of microseconds [16]) are applied to local variables $x_{i}, y_{i}$ and $\nu_{i}$ in series where variables currently not being updated are fixed to values evaluated immediately before. These updates form the decentralized algorithm listed in Alg. 1 for coordination of active nodes in Tier 3. Updates will be terminated once the primal and dual residuals $r$ and $s$ are below the threshold $\epsilon^{r}$ and $\epsilon^{s}$. The primal residual is the norm of the difference between the perspective and original variables and this is represented as $r_{i}^{k+1}=\left\|A_{i} x_{i}^{k+1}-B_{i} y_{i}^{k+1}\right\|$. Dual residual is expressed as $s_{i}^{k+1}=\left\|-\rho A_{i}^{T} B_{i}\left(y_{i}^{k+1}-y_{i}^{k}\right)\right\|$ and is associated with dual feasibility [31].

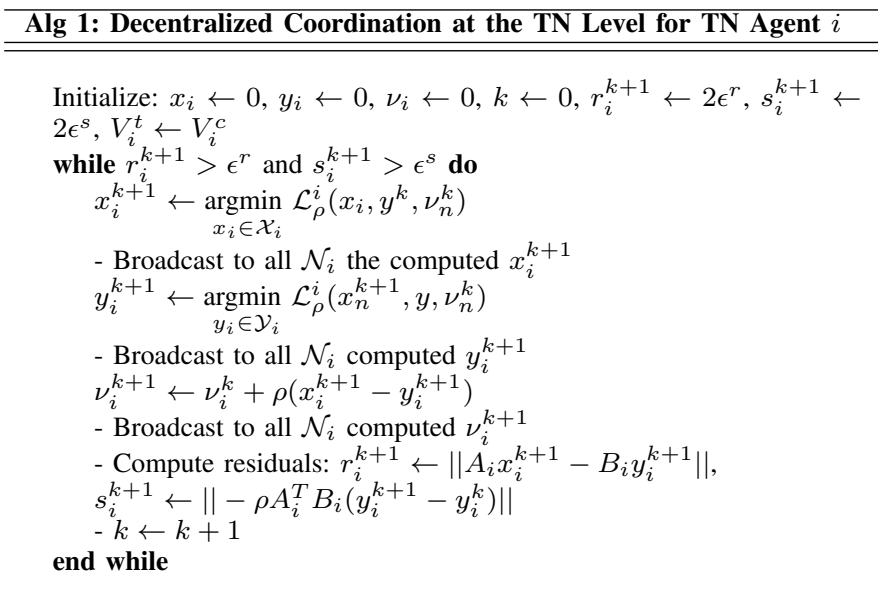

\section{COORdination IN TIER 2 AND TIER 1}

Tier 2 consists of agents that bridge co-ordination between the $\mathrm{TN}$ and elements (e.g. generation, DNs, etc.) that deliver or consumer power. Tier 1 is composed of actual nodes such as synchronous generation systems, IPPs and DNs that receive signals from Tier 2 agents to make local actuation decisions. Each one of these entities coordinate local power injections to achieve $p_{i}^{s}=p_{i}^{g}+p_{i}^{r}$ computed by TN agent $i$ in Tier 3 to which these are directly connected to over the subsequent 10 minute period. Coordinating power injections for large systems such as the DN that consists of thousands of residential consumers with a wide variety appliance mix, load curtailment preferences and sustainable micro-DGs is more challenging.

We consider real power actuation in Tier 1 (assume that reactive power supply is readily available from the main grid) for balanced single phase systems. In order to abstract from the actual elements coordinated in Tier 1, it is assumed that all Tier 1 entities have the same set of strategies $\mathcal{S}$ (power injection or power demand curtailment) at their disposal. These strategies are considered to be power changes with fixed magnitudes. Since revisions are made at random time instances, the impact of these are incremental from a system-wide perspective. For consistency, if a node has greater capacity than the strategies at hand, virtual agents are introduced. This allows us to apply population game theory regardless of the variations in the actuation capacities of individual nodes. For instance, if 
a large synchronous plant has a large generation capacity, then virtual local entities are created that can actuate from one of $S$ strategies. The number of virtual agents created is $\bar{p} / \max (\mathcal{S})$ where $\bar{p}$ is the maximum power actuation capacity. The signal computed by agent $i$ in Tier 2 is constructed by first formulating the general problem for local coordination:

$$
\begin{array}{r}
\left(\mathcal{P}_{T 2}^{i}\right) \quad \min _{x \in \triangle} f_{o}^{i}(x)=\sum_{j=1}^{n} C_{j} \cdot\left(m \cdot y_{j} \cdot x_{j}\right)^{2} \\
\text { s.t. } \sum_{j=1}^{n} m \cdot y_{j} \cdot x_{j}=p_{i}^{s}
\end{array}
$$

where $x_{j}$ is the proportion of agents in Tier 1 using strategy $j$, $C_{j}$ is the cost of strategy $j, n$ is the total number of strategies in $\mathcal{S}$ and $y_{j}$ is the power actuated in strategy $j$ and the coupling constraint dictates that the total power actuated (obtained from data concentrator in Tier 1) is equal to the power injection value $p_{j}^{s}$ computed by the corresponding TN agent in Tier 3 . The cost signal $F_{j}$ for strategy $j$ is selected to be the gradient of the Lagrangian of $\mathcal{P}_{T 2}$ :

$$
F_{j}(x)=\mathcal{K} \cdot m \cdot y_{j}\left(2 \cdot C_{j} m \cdot y_{j} \cdot x_{j}+\nu^{*}\right)
$$

As this is a convex optimization problem, the above cost signal transmitted by an agent in Tier 2 to local entities in Tier 1 will converge to zero given that there exists sufficient resources in the system to cumulatively achieve $p_{i}^{s}[28]$. Upon receiving this cost signal, Tier 1 entities will randomly select a time to respond using the probability $\rho_{m, k}(F)$ :

$$
\rho_{i, j}(F(x), x)=\frac{\left[F_{i}-F_{j}\right]_{+}}{n \cdot x_{i}}
$$

This is known as the projection revision in population game theory. The resulting system dynamic is:

$$
\dot{x}_{i}=\sum_{j=1}^{n} \rho_{j, i} \cdot x_{j}-x_{i} \cdot \sum_{j=1}^{n} \rho_{i, j}
$$

where the first term is the rate at which agents switch to strategy $i$ and the second term is the rate at which agents leave strategy $i$. This state dynamic is guaranteed to converge to the optimal solution as a Lyapunov function exists for the system dynamic due to the construction of the problem. As the cost function $F$ assigned to the strategies is the gradient of the Lagrangian of $\mathcal{P}_{T 2}$, the Lagrangian (i.e. potential function) is the Lyapunov function of the system dynamic [32]. When there exists sufficient system resources, the incremental effect of individual agents acting in a large population will result in the system dynamic $\dot{x}_{i}$ with guaranteed convergence to the optimal solution of $\mathcal{P}_{T 2}$.

Large populations of entities is natural in DNs and IPPs and this can be artificially constructed for large synchronous plants using virtual agents. Prior to revising local actuation, each agent in Tier 1 will ensure that this change in strategy heeds local generation capacity, available power injection within the local feeder and/or comfort requirements. This feasibility check is conducted by measuring the available power limits in the lines that connect the actuating entity to the main feeder.
Voltage and power flow in the distribution network can be modelled using the branch-flow model [11]:

$$
\begin{aligned}
& S_{i j}=S_{i}+\sum_{h \in \mathcal{C}_{i}}\left(S_{h i}-z_{h i} I_{h i}^{2}\right) \\
& \hat{V}_{i}-\hat{V}_{j}=2 \operatorname{Re}\left(z_{i j} S_{i j}\right)-\left|z_{i j}\right|^{2} I_{i j}^{2} \\
& I_{i j}^{2}=\frac{\left|S_{i j}\right|}{\hat{V}_{i}}
\end{aligned}
$$

where $S_{i j}=p_{i j}+\mathbf{j} q_{i j}$ is the complex power flow in line $i j, \mathbf{j}$ is the complex number $\sqrt{-1}, S_{i}$ is the complex power injection at bus $i, \hat{V}_{i}$ is the complex bus voltage magnitude squared, $z_{i j}$ is the line impedance and $\mathcal{C}_{i}$ is a set representing the immediate descendants of node $i$ from the perspective of the feeder node. The non-linear terms are associated with the resistive power losses $z_{h i} I_{h i}^{2}$. Ignoring these will result in a conservative approximation of voltage drop and power flow in the DN lines with negligible error [11]. During each strategy revision, the revising agent communicates the impending change in power injection to its ancestral nodes which will factor in the change in power flow to compute the approximate change in bus voltage magnitudes and power flow. If there is a violation in voltage magnitude limits or power flow, this is communicated to the revising node which will then halt the strategy revision. If no violations are encountered, then the agent will proceed with the strategy change. The complexity of this feasibility check is a constant which is the height of the radial network representing the DN.

If these conditions are not met, the strategy revision will not take place. These computations within Tier 1 and 2 levels are summarized in Alg. 2. System convergence based on this population game theory setup for Tiers 1 and 2 is independent of the number of Tier 1 agents participating in the system [32] and this will be shown under practical settings in Sec. VII.

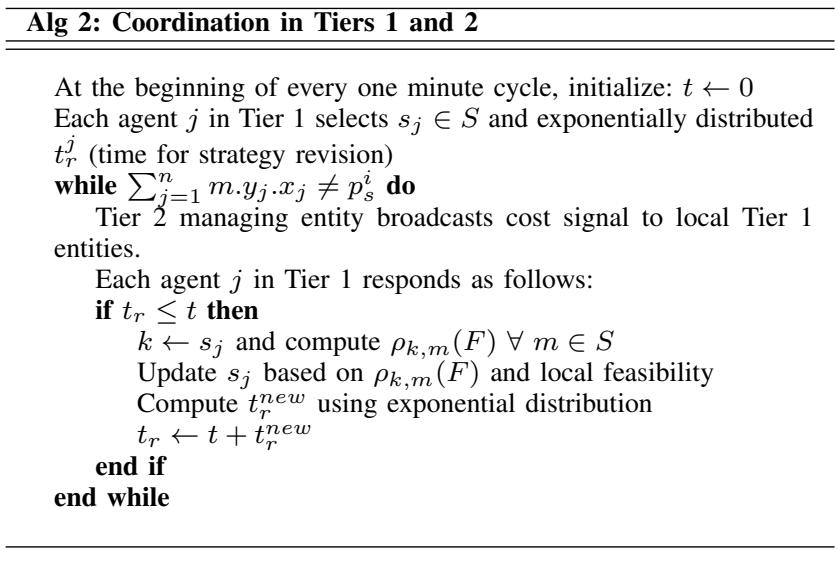

\section{Mathematical Tractability}

In this section, a discussion on the mathematical tractability of the proposed hierarchical grid management framework is presented. In Table II] the computational complexities of the proposed hierarchical method, centralized method applied to the linearized power-flow constraints presented in constraints (C1') - (C5'), (C3) and centralized optimization applied to 
the original non-convex optimization problem in $\mathcal{P}_{O P F}$ are compared. The Big-O notation is utilized to measure the asymptotic computational complexity for the three methods at the TN and DN levels separately and then these are combined.

ADMM is utilized in the proposed framework for coordination at the TN level. If the number of nodes at the TN level is $n$ and the optimization problem is convex, then the number of iterations required for the ADMM algorithm to converge is $\mathcal{O}(n)$ as established by reference [15]. At the DN level, broadcast signals are computed using population game theoretic constructs which are independent of the number of nodes present in the system [32]. Hence, the computational complexity at this level is $\mathcal{O}(k)$ where $k$ is a constant that is independent of the nodes present in the DN. Thus, the combined computational complexity for the overall proposed framework is $\mathcal{O}(k n)=\mathcal{O}(n)$.

Applying a centralized solver, such as the interior point method, to compute the optimal solution of a quadratic program with convex constraints results in computational complexity that is polynomial in the size of the problem [4]. This implies that the computational complexity at the TN level containing $n$ nodes is $\mathcal{O}\left(n^{p}\right)$ and at the $\mathrm{DN}$ level containing $m$ nodes is $\mathcal{O}\left(m^{p}\right)$. Combining the computational complexity at both levels results in $\mathcal{O}\left((m n)^{p}\right)$. It is clear that the computational complexity associated with the centralized solver is much more concentrated than the proposed hierarchical framework.

Finally, solving the original problem $\mathcal{P}_{O P F}$ for the $\mathrm{DN}$ and $\mathrm{TN}$ in a central manner is NP-hard due to the presence of a multitude of non-convex constraints that are associated with power flow balance at both the TN and DN levels. Thus, it is clear from these results that the hierarchical framework divides the monolithic and complex problem at hand into simpler manageable components that can be solved in a tractable manner by combining distributed and decentralized approaches.

TABLE II: Comparison of computational complexity.

\begin{tabular}{|l|c|c|c|}
\hline Tier & $\begin{array}{c}\text { Proposed } \\
\text { Hierarchical }\end{array}$ & $\begin{array}{c}\text { Centralized } \\
\text { Linear Constraints }\end{array}$ & $\begin{array}{c}\text { Centralized } \\
\text { Original OPF }\end{array}$ \\
\hline TN & $\mathcal{O}(n)$ & $\mathcal{O}\left(n^{p}\right)$ & NP-hard \\
DN & $\mathcal{O}(k)$ & $\mathcal{O}\left(m^{p}\right)$ & NP-hard \\
Combined & $\mathcal{O}(n)$ & $\mathcal{O}\left((n m)^{p}\right)$ & NP-hard \\
\hline
\end{tabular}

\section{IMPLEMENTATION}

In this section, the proposed hierarchical signal-processing framework is studied under realistic circumstances via practical simulations.

\section{A. Tier 3 Coordination}

In this section, the performance of the decentralized algorithm outlined in Alg. 1 for the Tier 3 level is evaluated in IEEE 39-bus, IEEE 118-bus and Polish 2736-bus systems. All line and bus parameters are obtained from MATPOWER. $V^{t}$ is set to 1 p.u. and the initial values of the optimization variables are set to 0 . First, the impact of $\rho$ on the convergence of the solution to optimality is evaluated in the IEEE 118-bus system in Fig. 3 for real power injection $P$. It is evident that the proportion of the computed value of $P_{\text {sim }}$ with respect to the actual solution $P_{a c t}$ varies significantly for different values of $\rho$. Based on these results, we have selected $\rho=100$ in the remaining simulations to ascertain reduced iterations.

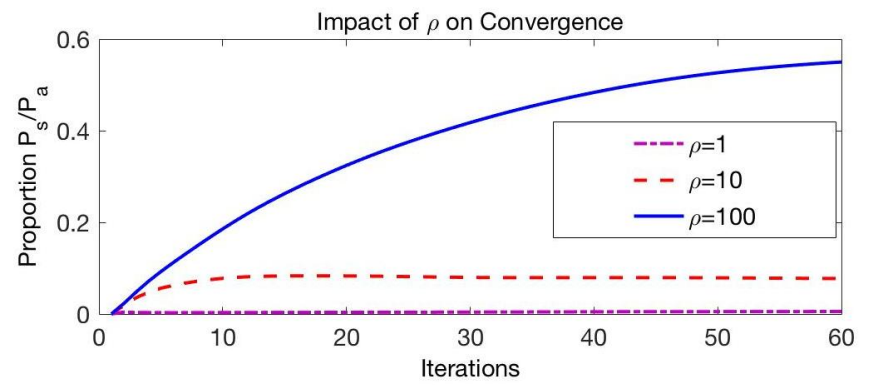

Fig. 3: Convergence in the IEEE 118-bus System.

Next, the relative root mean square error of optimization variable $x$ is averaged across the buses according to $x_{r m s e}=\left(\frac{1}{|\mathcal{B}|} \sum_{i \in \mathcal{B}}\left(\frac{x_{p}^{i}-x_{a}^{i}}{x_{a}^{i}}\right)^{2}\right)^{1 / 2}$ where $x_{p}^{i}$ is the result of the proposed algorithm and $x_{a}^{i}$ is the actual optimal solution obtained using the solver provided by MATPOWER. Results are presented in Table III for $x=P$ and $x=|V|$ in the IEEE 39-bus and IEEE 118-bus systems. Moreover, Table IV

TABLE III: Relative RMSE of Optimization Variables.

\begin{tabular}{|l|c|c|}
\hline & $\mathrm{P}_{\text {rmse }}$ & $\mathrm{V}_{\text {rmse }}$ \\
\hline IEEE 39-bus & 0.0055911 & 0.00050685 \\
IEEE 118-bus & 0.014166 & 0.004598 \\
\hline
\end{tabular}

contains specific comparison of power generation computed in the IEEE 118-bus system using the proposed decentralized technique and exact results obtained from MATPOWER in MegaWatts (MW) for the AC model. It is clear from these results that our decentralized proposal results in minor deviations that can be readily offset by ancillary services available in the TN.

TABLE IV: Real Power Injections in IEEE 118-bus System.

\begin{tabular}{|c|l|l|l|l|l|l|l|l|}
\hline Bus & $P_{\text {sim }}$ & $P_{a c t}$ & Bus & $P_{\text {sim }}$ & $P_{a c t}$ & Bus & $P_{\text {sim }}$ & $P_{\text {act }}$ \\
\hline 1 & 78.6 & 79.3 & 42 & 84.7 & 84.9 & 80 & 83.2 & 83.4 \\
4 & 78.3 & 78.7 & 46 & 88.9 & 84.4 & 85 & 78.6 & 78.5 \\
6 & 78.4 & 79.0 & 49 & 87.1 & 85.1 & 87 & 72.9 & 72.8 \\
8 & 78.3 & 78.7 & 54 & 86.1 & 86.7 & 89 & 79.4 & 78.6 \\
10 & 78.3 & 78.1 & 55 & 86.1 & 86.5 & 90 & 79.5 & 79.5 \\
12 & 78.3 & 79.7 & 56 & 86.1 & 86.6 & 91 & 79.7 & 77.7 \\
15 & 77.6 & 79.3 & 59 & 85.3 & 86.0 & 92 & 79.6 & 78.8 \\
18 & 77.2 & 78.7 & 61 & 84.9 & 84.5 & 99 & 78.2 & 78.7 \\
19 & 76.8 & 78.9 & 62 & 85.1 & 84.5 & 100 & 79.2 & 77.8 \\
24 & 74.5 & 76.5 & 65 & 84.3 & 83.5 & 103 & 72.1 & 74.9 \\
25 & 75.5 & 76.9 & 66 & 85.4 & 83.7 & 104 & 71.1 & 74.6 \\
26 & 75.5 & 77.2 & 69 & 82.2 & 82.8 & 105 & 70.8 & 74.3 \\
27 & 76.4 & 77.6 & 70 & 77.7 & 78.9 & 107 & 70.7 & 73.9 \\
31 & 76.8 & 77.7 & 72 & 72.6 & 74.9 & 110 & 67.9 & 71.2 \\
32 & 76.5 & 77.5 & 73 & 78.2 & 76.5 & 111 & 67.9 & 69.1 \\
34 & 81.2 & 80.9 & 74 & 81.4 & 81.2 & 112 & 67.9 & 71.0 \\
36 & 81.2 & 80.8 & 76 & 82.6 & 82.8 & 113 & 77.5 & 77.8 \\
40 & 82.8 & 83.5 & 77 & 82.9 & 83.6 & 116 & 83.6 & 83.3 \\
\hline
\end{tabular}

Results for the Polish 2736-bus system are not included 
in Table III as the size of the system and non-linearities in the original OPF formulation can result in inaccuracies in the solution computed by the built-in MATPOWER solver. Instead, the primal residual obtained with our proposal is plotted for the Polish 2736-bus system in Fig. 4 . It is clear that the residual decreases rapidly initially with minor differences later on as expected with the ADMM algorithm. Convergence for the Polish 2736-bus system occurs within 100 iterations. Communication delay incurred in signal exchanges is approximately $10 \mathrm{~ms}$ [16]. Thus, convergence will be achieved within 1 minute in the Polish 2736-bus system. As the coordination interval is 10 minutes in Tier 3 , there is a tolerance of communication delays in signal exchanges up to 9 minutes which accounts for uncertainties and synchronization issues of signals exchanged. The number of iterations needed for the IEEE 39-bus system and IEEE 118-bus system to reach the same residual is 20 and 28 iterations respectively. As expected, the size of the system has an impact on the convergence time of the system being coordinated. Theoretically, the convergence time of the ADMM algorithm is linear with respect to the size of the system for convex formulations [15].

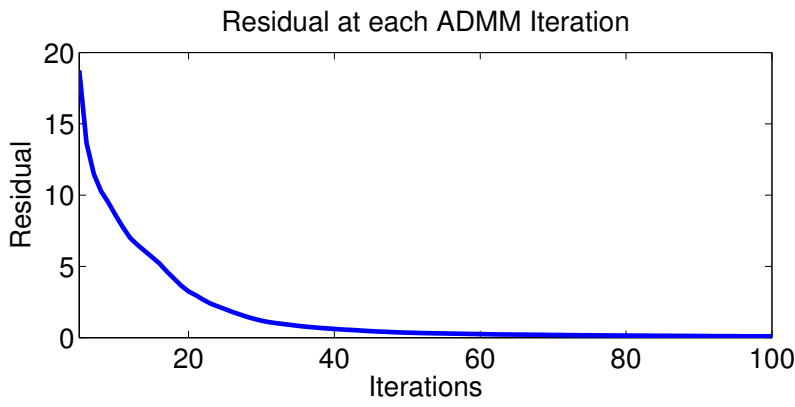

Fig. 4: Residual for the Polish 2736-bus System.

Next, ADMM is compared to other decentralized techniques such as dual decomposition (DD) and consensus algorithms used in the literature. DD can be applied to constrained optimization problems (e.g. [6], [14]). If the objective is separable, then the dual problem can be divided into subproblems that can be parallelized between multiple agents. Updates using the sub-gradient method are used to improve the overall solution. However, for guaranteed convergence, the original problem must be strictly convex. This is not the case for $\mathcal{P}_{O P F}^{d}$ as the objective is not a function of $V$ and $\Theta$. We applied the DD technique to $\mathcal{P}_{O P F}^{d}$ for many different constant step-size selected in the range of $\left[10^{-5}, 10^{3}\right]$ and were not able to observe convergence to the optimal solution as expected. The simulations had in fact diverged from optimality. With ADMM, this issue is eliminated by the augmented Lagrangian term that enforces strong convexity in the problem [31]. Consensus methods are also widely used in the literature to parallelize computations (e.g. [26]). However, the convergence speed is related to the second smallest eigenvalue of the Laplacian matrix representing the communication network topology. This, in the worst case, is proportional to $\mathcal{O}\left(n^{3}\right)$ as indicated by reference [33]. On the other hand, the convergence of the ADMM algorithm is linearly proportional to the size of the problem (i.e. $\mathrm{O}(n)$ ) [15]. Hence, even though this is a proof-of-concept, superior convergence properties of the ADMM method renders it suitable for decentralized management of buses in the TN as summarized in Table $\mathrm{V}$ This removes the need for a central coordinating entity at the TN level.

TABLE V: Comparison of Decentralized Techniques.

\begin{tabular}{|l|c|c|c|}
\hline & Parallel & Convergence Guarantee & Convergence Speed \\
\hline ADMM & $\checkmark$ & $\checkmark$ & $\mathrm{O}(n)$ \\
DD & $\checkmark$ & $x$ & N/A \\
Consensus & $\checkmark$ & $\checkmark$ & $\mathrm{O}\left(n^{3}\right)$ \\
\hline
\end{tabular}

\section{B. Tier 1 and 2 Coordination}

Based on the optimal power injection values computed in Tier 3, Tier 1 and Tier 2 coordination is exacted according to Alg. 2. To illustrate the effectiveness of Alg. 2 even in the
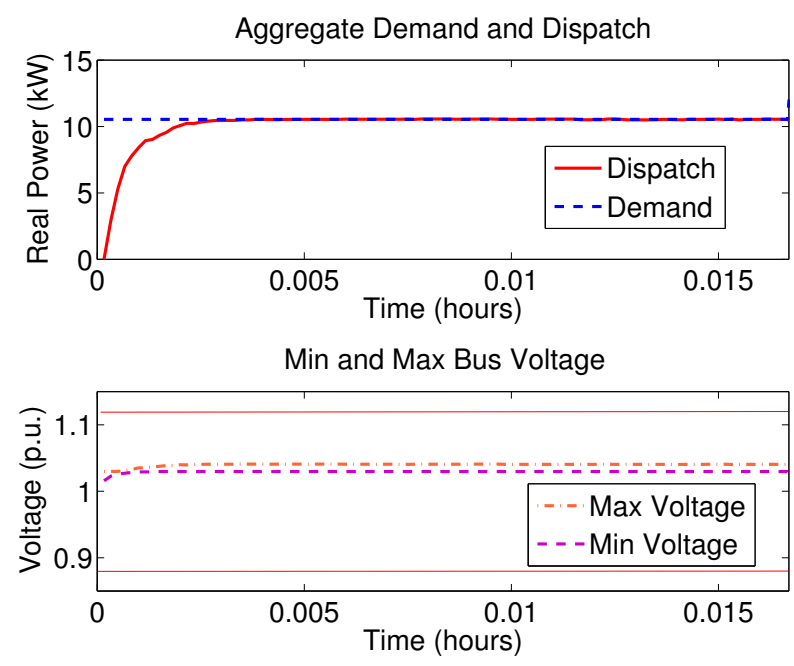

Fig. 5: Distributed Coordination in Danish DN.

presence of a large number of entities in Tier 1, the first result in this section illustrated in the second subplot of Fig. 5 depicts the rapid convergence of actuating DGs and flexible consumers totalling hundred entities residing in a Danish DN system with parameters obtained from [34] over one optimization interval (i.e. 1 minute) based on optimal power injection value computed in Tier 3 . The EPU is presiding as the managing entity in Tier 2. Fig. 6 illustrates the effect on bus voltages with no coordination scheme in place. Maximum voltages of

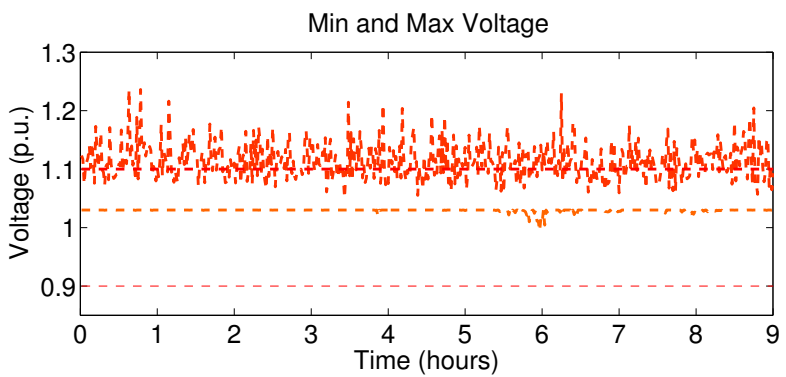

Fig. 6: Effect of no Coordination. 
buses in the DN are exceeding the 1.1 p.u. threshold. With coordination, this is not the case as illustrated in Fig. 5. Flexible consumers also conduct feasibility checks prior to making a demand curtailment decision. It is clear from Fig. 5 that the system of actuating components are able to achieve the power injection setpoint computed in Tier 3 exponentially fast and maintain voltages across the buses within feasible limits.

Thus, these results illustrate effectiveness of the hierarchical signal processing framework presented in this paper in allowing for the seamless, plug-and-play integration and coordination of diverse entities across the power grid.

\section{Performance Comparison with State-of-the-Art}

A large number of proposals in the existing literature aim to solve the OPF problem. In these proposals, the OPF is solved in its original form (i.e. non-convex) via heuristics or via convex relaxations which are applied for tractability. In the original non-convex form, although all original variables and constraints are accounted for, no guarantees on optimality and convergence can be established due to the presence of non-convex constraints. Iterative methods such as sub-gradient methods (e.g. dual decomposition), consensus methods and operator splitting methods (e.g. ADMM) fail as these algorithms can diverge due to the non-convexities in the problem formulation [24], [31]. Centralized solvers using heuristic techniques (e.g. genetic algorithm, simulated annealing, etc.) have been presented in the literature [35]. These result in sub-optimal solutions with no guarantees on the performance or convergence [12]. Moreover, these entail significant tuning of parameters based on the characteristics of the underlying system which can result in additional overhead. Finally, myopic techniques (e.g. droop control [36], machine learning [37], etc.) where nodes make decisions using only local measurements result in inefficiencies as the state of the external environment is not accounted in these decisions.

When relaxations are applied to the OPF, non-convex constraints are eliminated. For instance, DC power flow is heavily utilized by system operators in today's grids for planning purposes [4]. This formulation eliminates reactive power and bus voltage magnitudes which are important considerations in the integration of renewables and variable loads. Thus, the tradeoff for simplicity is essentially the practicality of the OPF formulation. Other types of relaxations such as semi-definite and second-order cone relaxations have been proposed for the DN [9]. These are well-suited for DNs that have tree topology and not for TNs which are associated with mesh structure [10]. Linear approximations of AC constraints presented in reference [23], which has been applied in this paper, retains all important variables and is applicable to high-voltage power networks with mesh topology like the TN [10].

Applying iterative methods to strictly convex optimization problems will guarantee convergence [26]. However, subgradient and consensus methods entail extensive parameter tuning (e.g. step-size) and are associated with polynomial convergence rates $\mathcal{O}\left(n^{p}\right)$ [6]. ADMM is associated with linear convergence rate $\mathcal{O}(n)$ with no need for fine-grained parameter tuning. Moreover, the underlying problem at hand is not required to be strictly convex for convergence (convexity suffices). Myopic decisions are independent of the OPF structure as these strive to maintain feasibility not optimality. A summary of the performance of various techniques applied in the state-of-the-art for different types of OPF formulations is presented in Table $\mathrm{VI}$

TABLE VI: Comparison of Optimal Performance.

\begin{tabular}{|l|c|c|c|}
\hline & $\begin{array}{c}\text { Strictly } \\
\text { Convex }\end{array}$ & Convex & Non-convex \\
\hline $\begin{array}{l}\text { Distributed/ } \\
\text { Decentralized }\end{array}$ & $\mathcal{O}\left(n^{p}\right)$ & N/A & Diverge \\
Sub-gradient & $\mathcal{O}\left(n^{p}\right)$ & $\mathcal{O}\left(n^{p}\right)$ & $\begin{array}{c}\text { Diverge } \\
\text { Consensus }\end{array}$ \\
Operator Splitting & $\mathcal{O}(n)$ & $\mathcal{O}(n)$ & Diverge \\
\hline $\begin{array}{l}\text { Centralized } \\
\text { Heuristics }\end{array}$ & $\begin{array}{c}\text { Sub-optimal } \\
\text { Quadratic Program }\end{array}$ & $\begin{array}{c}\text { Sub-optimal } \\
\mathcal{O}\left(n^{p}\right)\end{array}$ & $\begin{array}{c}\text { Sub-optimal } \\
\text { NP-Hard }\end{array}$ \\
\hline $\begin{array}{l}\text { Myopic } \\
\text { Droop control } \\
\text { Machine learning }\end{array}$ & $\begin{array}{c}\text { Not optimal } \\
\text { False } \\
\text { positives }\end{array}$ & $\begin{array}{c}\text { Not optimal } \\
\text { False } \\
\text { positives }\end{array}$ & $\begin{array}{c}\text { Not optimal } \\
\text { False } \\
\text { positives }\end{array}$ \\
\hline
\end{tabular}

\section{FINAL REMARKS}

In this paper, a hierarchical signal processing framework is presented which allows for the effective and sustainable coordination of a diverse set of power components across the modern grid while ensuring that the grid limits imposed on the underlying complex physical network structure are taken into account. With abstraction and decomposition built into decision-making at each tier of the proposed framework, every actuating node is able to adapt to changing conditions across the entire grid. This ability to respond in an adaptive manner not only enhances the resilience of the grid but also enables a large number of heterogeneous power components to seamlessly interact with the grid in a unified and nondisruptive manner. In future work, we hope to investigate myopic decision-making based solely on local measurement signals to address potentially disruptive transient conditions in microgrid settings into our hierarchical framework.

\section{REFERENCES}

[1] Future of the Grid, Gridwise Alliance. [Online]. Available at: http://energy.gov/oe/downloads/ future-grid-evolving-meet-america-s-needs-december-2014] [Accessed: 21-Jan-2016].

[2] C. L. Masters, "Voltage rise the big issue when connecting embedded generation to long $11 \mathrm{kV}$ overhead lines," Journal of Power Engineering, vol. 16, no. 1, pp. 5-12, Feb. 2002.

[3] J. D. Glover, T. J. Overbye, and M. S. Sarma, Power system analysis \& design. Boston, MA: Cengage Learning, 2017.

[4] J. Taylor. Convex Optimization of Power Systems. Cambridge University Press, 2015.

[5] K. G. Murty and S. N. Kabadi, "Some NP-complete problems in quadratic and nonlinear programming," Mathematical Programming, vol. 39, no. 2, pp. $117129,1987$.

[6] J. Joo and M.D. Ilic. Multi-layered optimization of demand resources using lagrange dual decomposition. IEEE Transactions on Smart Grid, 4(4):2081-2088, 2013.

[7] Z. Yang, H. Zhong, Q. Xia, and C. Kang, "A novel network model for optimal power flow with reactive power and network losses," Electric Power Systems Research, vol. 144, pp. 6371, 2017. 
[8] Z. Yang, H. Zhong, A. Bose, T. Zheng, Q. Xia, and C. Kang, "A Linearized OPF Model with Reactive Power and Voltage Magnitude: A Pathway to Improve the MW-Only DC OPF," IEEE Transactions on Power Systems, pp. 111, 2017.

[9] S.H. Low. Convex Relaxation of Optimal Power Flow Part I: Formulations and Equivalence. IEEE Transactions on Control of Network Systems, 1(1):15-27, 2014.

[10] S.H. Low. Convex Relaxation of Optimal Power Flow Part II: Exactness. IEEE Transactions on Control of Network Systems, 1(2):177-189, 2014.

[11] Masoud Farivar and Steven H. Low. "Branch Flow Model: Relaxations and Convexification (Parts I, II)". IEEE Transactions on Power Systems, 28(3):2554-2572, August 2013.

[12] S. Diamond, R. Takapoui, S. Boyd, "A general system for heuristic solution of convex problems over nonconvex sets", Jan. 2016

[13] A. Finamore, V. Galdi, V. Calderaro, A. Piccolo, G. Conio, and S. Grasso, "Artificial neural network application in wind forecasting: an one-hour-ahead wind speed prediction," IET International Conference on Renewable Power Generation 2016.

[14] P. Srikantha and D. Kundur. Distributed optimization of dispatch in sustainable generation systems via dual decomposition. IEEE Transactions on Smart Grid, 6(5):2501 - 2509, 2015.

[15] Q. Peng and S. Low. Distributed algorithm for optimal power flow on a radial network. IEEE Conference on Decision and Control, 2014.

[16] V. Gungor, D. Sahin, T. Kocak, S. Ergut, C. Buccella, C. Cecati, G. Hancke and D. Kundur. "Smart Grid Technologies: Communication Technologies and Standards, IEEE Transactions on Industrial Informatics, vol. 7, no. 4, pp. 529-539, 2011.

[17] M. D. Ilic, "From Hierarchical to Open Access Electric Power Systems," Proceedings of the IEEE, vol. 95, no. 5, pp. 10601084, 2007.

[18] D. Lee, P. Srikantha, and D. Kundur, "Secure operating region simplification in dynamic security assessment," IEEE International Conference on Smart Grid Communications, 2015

[19] X. Hu, H. Zhou, Z.-W. Liu, X. Yu, and C. Li, "Hierarchical Distributed Scheme for Demand Estimation and Power Reallocation in a Future Power Grid," IEEE Transactions on Industrial Informatics, vol. 13, no. 5, pp. 2279-2290, 2017

[20] R. J. Sanchez-Garcia, M. Fennelly, S. Norris, N. Wright, G. Niblo, J. Brodzki, and J. W. Bialek, "Hierarchical Spectral Clustering of Power Grids," IEEE Transactions on Power Systems, vol. 29, no. 5, pp. 22292237, 2014.

[21] W. Feng, K. Sun, Y. Guan, J. Guerrero, and X. Xiao, "Active Power Quality Improvement Strategy for Grid-connected Microgrid Based on Hierarchical Control,” IEEE Transactions on Smart Grid, pp. 1-1, 2016.

[22] P. Tian, X. Xiao, K. Wang, and R. Ding, "A Hierarchical Energy Management System Based on Hierarchical Optimization for Microgrid Community Economic Operation," IEEE Transactions on Smart Grid, vol. 7, no. 5, pp. 2230-2241, 2016.

[23] C. Coffrin and P. V. Hentenryck, "A Linear-Programming Approximation of AC Power Flows," INFORMS Journal on Computing, vol. 26, no. 4, pp. 718734, 2014.

[24] T. Erseghe, "Distributed Optimal Power Flow Using ADMM," IEEE Transactions on Power Systems, vol. 29, no. 5, pp. 2370-2380, 2014.

[25] S. Bolognani, R. Carli, G. Cavraro, and S. Zampieri. A distributed control strategy for optimal reactive power flow with power and voltage constraints. IEEE Smart Grid Communications, pages 115-120, 2013.

[26] W. Zhang, W. Liu, X. Wang, L. Liu, and F. Ferrese. Online optimal generation control based on constrained distributed gradient algorithm. IEEE Transactions on Power Systems, 30(1):35-45, 2015.

[27] P. Srikantha and D. Kundur, "Resilient Distributed Real-Time Demand Response via Population Games," IEEE Transactions on Smart Grid, pp. 112, 2016.

[28] P. Srikantha and D. Kundur, "Real-Time Integration of Intermittent Generation with Voltage Rise Considerations," IEEE Transactions on Sustainable Energy, pp. 11, 2016.

[29] D. Lee, P. Srikantha, and D. Kundur, "Secure operating region simplification in dynamic security assessment," IEEE International Conference on Smart Grid Communications, 2015.

[30] E. Wei and A. Ozdaglar, "On the o $(1 / \mathrm{k})$ convergence of asynchronous distributed alternating direction method of multipliers," arXiv preprint arXiv:1307.8254, 2013.

[31] S. Boyd, "Distributed Optimization and Statistical Learning via the Alternating Direction Method of Multipliers," Foundations and Trends in Machine Learning, vol. 3, no. 1, pp. 1122, 2010.
[32] W. H. Sandholm, Population Games and Evolutionary Dynamics. Cambridge, MA: MIT Press, 2011.

[33] A. Olshevsky and J. N. Tsitsiklis, "Convergence Speed in Distributed Consensus and Averaging," SIAM Review, vol. 53, no. 4, pp. 747772, 2011.

[34] J. Pillai, P. Thgersen, J. Mller, and B. Bak-Jensen, "Integration of electric vehicles in low voltage danish distribution grids," IEEE Power and Energy Society General Meeting, pages 1-8, 2012.

[35] A. Bakirtzis, P. Biskas, C. Zoumas, and V. Petridis, "Optimal power flow by enhanced genetic algorithm," IEEE Transactions on Power Systems, vol. 17, no. 2, pp. 229-236, 2002.

[36] P. Vovos, A. Kiprakis, A. Wallace, and G. Harrison. Centralized and distributed voltage control: Impact on distributed generation penetration. IEEE Transactions on Power Systems, 22(1):476-483, 2007.

[37] R.R. Negenborn, A.G. Beccuti, T. Demiray, S. Leirens, G. Damm, B. De Schutter, and M. Morari, "Supervisory hybrid model predictive control for voltage stability of power networks," American Control Conference, 2007

[38] S. Kanna, D. H. Dini, Y. Xia, S. Y. Hui, and D. P. Mandic, ?Distributed widely linear Kalman filtering for frequency estimation in power networks,? IEEE Trans. Signal, Inform. Process. Netw., vol. 1, no. 1, pp. 45-57, 2015.

[39] [2] H. J. Liu, W. Shi, and H. Zhu, ?Decentralized dynamic optimization for power network voltage control,? IEEE Trans. Signal, Inform. Process., Netw., vol. 3, no. 3, pp. 568-579, 2017. 